TRANSACTIONS OF THE

AMERICAN MATHEMATICAL SOCIETY

Volume 315, Number 2, October 1989

\title{
VALUE FUNCTIONS ON CENTRAL SIMPLE ALGEBRAS
}

\author{
PATRICK J. MORANDI
}

\begin{abstract}
In this paper we study noncommutative valuation rings as defined by Dubrovin. While there is in general no valuation associated to a Dubrovin valuation ring, we show that there is a value function associated to any Dubrovin valuation ring integral over its center. By using value functions we obtain information on Dubrovin valuation rings in a tensor product, both generalizing and giving a much simpler proof of a result about valued division algebras. By being able to work directly with central simple algebras we gain new information about division algebras over Henselian fields.
\end{abstract}

\section{INTRODUCTION}

In recent years the study of valuations on division algebras has proven useful in solving problems in the theory of algebras. For instance, valued division algebras were used in the construction of noncrossed products $\left[\mathrm{A}, \mathrm{JW}_{1}, \mathrm{~T}\right]$ and in the construction of division algebras with nontrivial $S K_{1}[\mathrm{P}, \mathrm{DK}]$. The Malcev-Neumann algebras studied by Tignol and Amitsur in [TA] are further examples of valued division algebras. Work on valued division algebras has intensified somewhat in the last few years, and a reasonable amount is known about such algebras (see for instance [ $\left.\mathrm{JW}_{2}, \mathrm{PY}, \mathrm{TW}\right]$ ).

There are some problems in dealing with valued division algebras. One problem in particular is how to generalize the notion of valuation to matrix algebras. Matrix algebras arise naturally when dealing with division algebras, for instance as the tensor product of two division algebras. Having a "valuation" on a matrix algebra that yields information about the underlying division algebra of the algebra would be a helpful tool, since getting at the underlying division algebra can be quite difficult in general. In Van Geel's book [V] there are a number of valuation ring and valuation-like conditions. A further candidate for valuation rings in matrix algebras is the rings Dubrovin defined in $\left[D_{1}\right]$. The nice structure and good extension and uniqueness properties of Dubrovin valuation rings (see $\left.\left[\mathrm{W}_{1}\right]\right)$ indicate that these rings are perhaps the correct definition of a valuation ring of a simple Artinian ring.

Received by the editors February 22, 1989.

1980 Mathematics Subject Classification (1985 Revision). Primary 13A18, 16A39.

This paper constitutes part of the author's doctoral dissertation. This author was supported as a research assistant by the N.S.F. during his final year of graduate school. 
In this paper we introduce the notion of a value function on a simple Artinian ring. While there is no value function associated to an arbitrary Dubrovin valuation ring, we will prove that there is a value function associated to a Dubrovin valuation ring which is integral over its center. Value functions behave similarly to valuations on division algebras. We will prove a number of results about integral Dubrovin valuation rings (that is, Dubrovin valuation rings integral over their center) by using value functions in much the same way as one would use a valuation. For instance, by using value functions we are able to get information about Dubrovin valuation rings in a tensor product (Theorem 3.3). This leads to new information about division algebras over Henselian fields (see Corollary 3.6 and the comments preceding it). In [MW] this author and A. Wadsworth will use value functions to study valued division algebras. By being able to work directly with matrix algebras we will give new and simpler proofs of some results in $\left[\mathrm{JW}_{2}\right]$. For the rest of this section we will give some definitions and elementary properties.

Let $D$ be a division algebra with $F=Z(D)$, the center of $D$. We will only be considering finite dimensional algebras, i.e. $[D: F]<\infty$. A subring $R$ of $D$ is called an invariant valuation ring of $D$ provided that for all $d \in D^{*}$,

(a) $d \in R$ or $d^{-1} \in R$,

(b) $d R d^{-1}=R$.

We will denote by $T^{*}$ the group of units of a ring $T$. Property (b) shows that any one-sided ideal of $R$ is actually two-sided, and (a) shows that the ideals of $R$ are linearly ordered by inclusion. Thus the set $\Gamma=\left\{d R \mid d \in D^{*}\right\}$ can be made into a totally ordered group by setting $d R \cdot e R=d e R$ and $d R \leq e R$ iff $d R \supseteq e R$. Invariant valuation rings are those rings which arise from a valuation on $D$. A valuation on $D$ is a function $v: D^{*} \rightarrow \Delta$, where $\Delta$ is a totally ordered abelian group, such that for all $a, b \in D^{*}$,

(1) $v(a b)=v(a)+v(b)$,

(2) $v(a+b) \geq \min \{v(a), v(b)\}$ if $a+b \neq 0$.

This is the definition given in Schilling's book [S]. For convenience we extend $v$ to $D$ by setting $v(0)=\infty$, where $\infty>\delta$ and $\infty+\delta=\delta+\infty=\infty$ for all $\delta \in \Delta$. The connection between valuations and invariant valuation rings is as follows. If $v$ is a valuation on $D$ then $R_{v}=\{d \in D \mid v(d) \geq 0\}$ is easily seen to be an invariant valuation ring. Conversely, if $R$ is an invariant valuation ring of $D$, then the map $v: D^{*} \rightarrow \Gamma$ given by $v(d)=d R$ for $\Gamma$ as above is a valuation on $D$.

Suppose $v$ is a valuation on $D$ with corresponding invariant valuation ring $R$. Then $R^{*}=\operatorname{ker}(v)$, so $D^{*} / R^{*} \cong v\left(D^{*}\right)$. We call $D^{*} / R^{*}$ the value group of $R$ (or $v$ ) and denote it $\Gamma_{R}$. The Jacobson radical $J(R)$ of $R$ is the set $\left\{d \in R \mid d=0\right.$ or $\left.d^{-1} \notin R\right\}=R-R^{*}$, so $J(R)$ is the unique maximal left (and right) ideal of $R$. Thus $\bar{R}=R / J(R)$ is a division ring, called the residue division ring of $R$. 
As is well known (e.g. [E, 7.1, 8.6]) for commutative fields the notions of valuation, valuation ring and place are all equivalent. Dubrovin's rings correspond to the idea of a place on a simple Artinian ring. These rings are defined as follows. A subring $B$ of a simple Artinian ring $S$ is called a Dubrovin valuation ring of $S$ provided that there is an ideal $J$ of $B$ such that

(a) $B / J$ is simple Artinian,

(b) If $s \in S-B$ then there are $b_{1}, b_{2} \in B$ with $b_{1} s, s b_{2} \in B-J$.

The place associated to $B$ is the map $S \rightarrow B / J \cup\{\infty\}$ given by $s \mapsto s+J$ if $s \in B$ and $s \mapsto \infty$ otherwise. It turns out that $J=J(B)$ and that $B$ is a twosided order in $S$. Thus $B$ is a prime Goldie ring. Furthermore, if $F=Z(S)$ then $Z(B)=B \cap F$ is a valuation ring of $F$. Dubrovin proved the following properties, which justify calling these rings valuation rings.

(1) The two-sided ideals of $B$ are linearly ordered by inclusion, although the one-sided ideals are generally not linearly ordered.

(2) Finitely generated one-sided ideals are principal and projective as $B$ modules.

(3) If $A$ is an overring of $B$ in $S$, then $A$ is a Dubrovin valuation ring of $S$ and $J(A)$ is a prime ideal of $B$. If $[S: F]<\infty$ then there is a 1-1 correspondence between the prime ideals of $B$, prime ideals of $Z(B)$ and overrings of $B$ in $S$. Furthermore if $P=J(A) \cap Z(B)$, then $A=B_{P}$, the central localization of $B$ at $P$.

(4) If $C$ is a subring of $B$ containing $J(B)$ then $C$ is a Dubrovin valuation ring of $S$ iff $C / J(B)$ is a Dubrovin valuation ring of $B / J(B)$.

(5) The $n \times n$ matrix ring $M_{n}(B)$ is a Dubrovin valuation ring in $M_{n}(S)$. Conversely, if $A$ is a Dubrovin valuation ring of $M_{n}(S)$ then there is a Dubrovin valuation ring $A_{0}$ of $S$ and an $x \in M_{n}(S)^{*}$ with $A=x M_{n}\left(A_{0}\right) x^{-1}$.

Proofs of these statements can be found in $\left[D_{1}, \S 1\right.$, Theorems 4 and 7; §2, Theorem 4] and [ $\mathrm{D}_{2}, \S 1$, Proposition 2; $\S 2$, Theorem 1]. Examples of Dubrovin valuation rings include invariant valuation rings, matrices over invariant valuation rings and Azumaya algebras over commutative valuation rings $\left[D_{2}, \S 2\right.$, Proposition 1]. If $V$ is a discrete valuation ring of a field $F$ and $S$ is central simple $F$-algebra then a subring $B$ of $S$ is a Dubrovin valuation ring of $S$ with $B \cap F=V$ iff $B$ is a maximal order over $V$ [W ${ }_{2}$, Ex. 1.15]. The literature on Dubrovin valuation rings consists of the papers $\left[D_{1}, D_{2}, B G, W_{1}\right.$ and $\left.\mathrm{W}_{2}\right]$.

For a Dubrovin valuation ring $B$ of $S$, there is a residue ring $\bar{B}=B / J(B)$, a simple Artinian ring. Furthermore, as in [ $\mathrm{W}_{2}$, p. 304], we can define a value group. If $s t(B)=\left\{s \in S^{*} \mid s B s^{-1}=B\right\}$ then $s t(B)$ is the normalizer of $B^{*}$ in $S^{*}$. We then define the value group $\Gamma_{B}=s t(B) / B^{*}$. Dubrovin proves in $\left[\mathrm{D}_{2}\right.$, $\S 2$, Proposition 4] that the monoid of two-sided ideals of $B$ is commutative, hence $\Gamma_{B}$ is an abelian group. If $S$ is a division ring and $B$ an invariant valuation ring of $S$ then $s t(B)=S^{*}$, so this definition of $\Gamma_{B}$ is the same as the one given above. If $F=Z(S)$ and $V=B \cap F$ then as noted above $V$ is a 
valuation ring of $F$. It is easy to see that $J(B) \cap V=J(V)$ and $B^{*} \cap V=V^{*}$. Thus there are natural inclusions $\Gamma_{V} \subseteq \Gamma_{B}$ and $\bar{V} \subseteq Z(\bar{B})$.

There are two integer invariants $t_{B}$ and $n_{B}$ which help to understand and classify $B$. For example, $n_{B}=t_{B}$ iff $B$ is integral over $V\left[\mathrm{~W}_{2}\right.$, Theorem F]. They are defined as follows. First, since $\bar{B}$ is simple Artinian, by Wedderburn's theorem, $\bar{B}$ is a matrix ring over a division ring. Let $t_{B}$ be the matrix size of $\bar{B}$. For $n_{B}$, if $\left(F_{h}, V_{h}\right)$ is the Henselization of $(F, V)$, then $n_{B}$ is the matrix size of $S \otimes_{F} F_{h}$. Wadsworth proves in [W $\mathrm{W}_{2}$, Theorem D] that $t_{B}$ divides $n_{B}$ and gives a formula for $s_{B}=n_{B} / t_{B}$ which lends itself to calculation. Furthermore, there is a version of the "Ostrowski theorem" $\left[\mathrm{W}_{2}\right.$, Theorem $\left.\mathrm{C}\right]$ :

$$
[S: F]=\left|\Gamma_{B}: \Gamma_{V}\right|[\bar{B}: \bar{V}] s_{B}^{2} \delta(B)
$$

where $\delta(B)$ is called the defect of $B$ and is 1 if $\operatorname{char}(\bar{V})=0$ and is a power of $\operatorname{char}(\bar{V})$ when this characteristic is prime. $B$ is said to be defectless when $\delta(B)=1$. We sometimes write $\delta(B / V)$ for $\delta(B)$ to indicate what the base ring is. As in the commutative (and invariant) case we call $e_{B, V}=\left|\Gamma_{B}: \Gamma_{V}\right|$ the ramification index and $f_{B, V}=[\bar{B}: \bar{V}]$ the residue degree of $B / V$. It is proven in $\left[\mathrm{M}_{1}\right.$, Theorem 2] that $B$ is invariant iff $n_{B}=1$, and so (1) reduces to the Ostrowski theorem proved in [ $\mathrm{M}_{1}$, Theorem 3] and in [Dr, Theorem 2] for $F$ Henselian.

For commutative valuation rings and invariant valuation rings a significant use of the value group is to classify the ideals. If $R$ is an invariant valuation ring of $D$ then the elements of $\Gamma_{R}$ are in 1-1 correspondence with the principal ideals of the form $d R$ for $d \in D^{*}$. Since all $R$-ideals (that is $R-R$ submodules of $D$ ) are sums of ideals of the form $d R$, as $d R=R d, \Gamma_{R}$ determines all $R$-ideals. For a Dubrovin valuation ring $B$ of $S$ this reasoning no longer holds since it is not true that any one-sided ideal of $B$ is two-sided. The elements of $\Gamma_{B}$ are in 1-1 correspondence with the ideals of the form $s B=B s$ (so $s \in$ $s t(B)$ ), and so $\Gamma_{B}$ classifies those $B$-ideals of the form $\sum_{s \in \mathscr{T}} s B$, where $\mathscr{T}$ is a subset of $\operatorname{st}(B)$. In general, not all $B$-ideals are of this form; indeed, all $B$-ideals are of this form precisely when $B$ is integral over its center $\left[\mathrm{M}_{2}\right.$, Theorem 3.1 or $\mathrm{W}_{2}$, Theorem F]. For $B$ integral this property of $B$-ideals is what allows us to define a value function on $S$ (see Definition 2.1). Integral Dubrovin valuation rings seem to behave the most like invariant valuation rings, and the presence of a value function allows many results about invariant valuation rings to be carried over to integral Dubrovin valuation rings.

One way Dubrovin valuation rings can be used to study valued division algebras is to help answer tensor product questions. For instance, suppose $D_{1}$ and $D_{2}$ are $F$-central division algebras with $R_{i} \subset D_{i}$ invariant valuation rings and $R_{1} \cap F=R_{2} \cap F=V$. Since $D_{1} \otimes_{F} D_{2}$ is a central simple $F$-algebra, $D_{1} \otimes_{F} D_{2} \cong M_{n}(D)$ for some $F$-central division algebra $D$. Suppose $D$ contains an invariant valuation ring $R$ with $R \cap F=V$. In general, getting at $R$ can be quite difficult. However, $B=M_{n}(R)$ is a Dubrovin valuation ring of 
$D_{1} \otimes_{F} D_{2}$ with $\Gamma_{B}=\Gamma_{R}$ and $\bar{B}=M_{n}(\bar{R})$ [W ${ }_{2}$, Corollary 3.5]. In a number of occasions in [MW] we construct a Dubrovin valuation ring $B^{\prime}$ in $D_{1} \otimes_{F} D_{2}$ with $B^{\prime} \cap F=V$ without too much difficulty. The following theorem, proved by Brungs and Gräter [BG, Theorem 5.4] for $V$ of finite rank and by Wadsworth $\left[\mathrm{W}_{2}\right.$, Theorem $\left.\mathrm{A}\right]$ in general shows $B^{\prime} \cong M_{n}(R)$.

Theorem 1.1. If $S$ is a central simple $F$-algebra with $B_{1}$ and $B_{2}$ Dubrovin valuation rings of $S$ and $B_{1} \cap F=B_{2} \cap F=V$, then $B_{2}=s B_{1} s^{-1}$ for some $s \in S^{*}$.

Thus we can determine $\Gamma_{R}$ and $\bar{R}$ by determining $B^{\prime}$. In this paper and in [MW] such a $B^{\prime}$ is found by observing that on $D_{1} \otimes_{F} D_{2}$ there is a naturally occurring value function which gives rise to $B^{\prime}$ just as a valuation gives rise to an invariant valuation ring.

\section{VAlUe FUnCtions}

In this section we will define value functions and prove some basic properties. The results of the next section and [MW] will make heavy use of value functions.

Definition 2.1. Let $S$ be a simple Artinian ring and $\Gamma$ a totally ordered abelian group. A function $w: S \rightarrow \Gamma \cup\{\infty\}$ is called a value function on $S$ provided that for all $s, t \in S$,

(1) $w(s)=\infty$ iff $s=0$ and $w(-1)=0$,

(2) $w(s+t) \geq \min \{w(s), w(t)\}$,

(3) $w(s t) \geq w(s)+w(t)$,

(4) $\operatorname{im}(w)=w(s t(w))$, where st $(w)=\left\{s \in S^{*} \mid w\left(s^{-1}\right)=-w(s)\right\}$.

The above definition was motivated by the following example: let $D$ be a division algebra and $v$ a valuation on $D$ with valuation ring $R$. If $S=M_{n}(D)$, we define a function $w: S \rightarrow \Gamma_{R} \cup\{\infty\}$ by

$$
w\left(\left(d_{i j}\right)\right)=\min _{i, j}\left\{v\left(d_{i j}\right)\right\} .
$$

It is easy to see that $w$ satisfies the conditions of Definition 2.1. This type of example can be found in [K]. By using value functions Kupferoth obtains a valuation on the universal division algebra $U D(\mathbf{Q}, n, t)$. It was recently pointed out to us that Benz studied value functions with value group a subgroup of $\mathbf{R}$ in $[\mathrm{Be}]$.

The difference between value functions and valuations shows up in condition (3) of the above definition. This less restrictive multiplicative condition allows matrix algebras to have value functions, as seen by the above example. While it would appear that (3) is not a very restrictive condition, we shall see in Theorem 2.4 and Proposition 2.6 that this is not the case, and that equality in (3) holds sufficiently often for value functions to be an effective tool.

Lemma 2.2. Let $w$ be a value function on $S$. Then

(1) If $s \in s t(w)$ and $t \in S$ then $w(s t)=w(t s)=w(s)+w(t)$. 
(2) $s t(w)$ is a subgroup of $S^{*}$ and $w: s t(w) \rightarrow \Gamma$ is a homomorphism.

(3) If $w(s) \neq w(t)$ then $w(s+t)=\min \{w(s), w(t)\}$.

(4) $B_{w}=\{s \in S \mid w(s) \geq 0\}$ is a ring and $J_{w}=\{s \in S \mid w(s)>0\}$ is a two-sided ideal of $B_{w}$.

Proof. As $w(t)=w\left(s^{-1} s t\right) \geq w\left(s^{-1}\right)+w(s t) \geq-w(s)+w(s)+w(t)=w(t)$, we see equality holds everywhere, giving (1). If $s, t \in s t(w)$ then $w(t)=-w\left(t^{-1}\right)$, showing $t^{-1} \in s t(w)$. Then by (1), w( $\left.(s t)^{-1}\right)=w\left(t^{-1} s^{-1}\right)=w\left(t^{-1}\right)+w\left(s^{-1}\right)=$ $-w(t)-w(s)=-(w(s)+w(t))=-w(s t)$. Thus $s t(w)$ is a subgroup of $S^{*}$ and by (1) again, $w: s t(w) \rightarrow \Gamma$ is a homomorphism.

For (3) we first note that $-1 \in \operatorname{st}(w)$ since $w\left((-1)^{-1}\right)=w(-1)=0=$ $-w(-1)$. Thus for all $s \in S, w(-s)=w(-1)+w(s)=w(s)$ by (1). Now if $w(s)>w(t)$ then $w(t)=w((s+t)+(-s)) \geq \min \{w(s+t), w(s)\}$. Since $w(s)>w(t)$, this implies $w(t) \geq w(s+t) \geq \min \{w(s), w(t)\}=w(t)$, giving $w(s+t)=\min \{w(s), w(t)\}$.

Finally, to prove (4), properties (2) and (3) of Definition 2.1 show $B_{w}$ is closed under addition and multiplication, and since $w(-s)=w(s)$ for all $s \in$ $S, B_{w}$ is closed under subtraction. Furthermore, $1 \in B_{w}$ as $w(-1)=0$. Therefore $B_{w}$ is a subring of $S$. Using properties (2) and (3) again, $J_{w}$ is seen to be a two-sided ideal of $B_{w}$.

If $R$ is an invariant valuation ring of a division algebra $D$ then there is a valuation $v$ on $D$ with $R=\{d \in D \mid v(d) \geq 0\}$ and $J(R)=\{d \in D \mid v(d)>$ $0\}$. The next theorem shows the existence of a value function on a simple Artinian ring where $B_{w}$ (as in Lemma 2.2) is a Dubrovin valuation ring.

Theorem 2.3. Let $S$ be a simple Artinian ring and $B$ a Dubrovin valuation ring of $S$. Suppose for all $s \in S-\{0\}$ there is an $\alpha \in \operatorname{st}(B)$ with $B s B=\alpha B$. Then there is a value function $w$ on $S$ with $B=B_{w}$ and $J(B)=J_{w}$. Furthermore, for any such $w$, st $(w)=\operatorname{st}(B), B^{*}=\{s \in \operatorname{st}(B) \mid w(s)=0\}$ and $w$ induces an isomorphism $\Gamma_{B} \rightarrow \operatorname{im}(w)$.

Proof. We define a value function $w: S \rightarrow \Gamma_{B} \cup\{\infty\}$ as follows. First, let $w(0)=\infty$. For $s \in S-\{0\}$, by hypothesis there is an $\alpha \in \operatorname{st}(B)$ with $B s B=$ $\alpha B$. Let $w(s)=\alpha B^{*} \in \Gamma_{B}$, i.e. $w(s)$ is the image of $B s B$ in $\Gamma_{B}$. It is clear that $w$ is well defined since $\alpha B=\beta B$ iff $\alpha B^{*}=\beta B^{*}$ in $\Gamma_{B}$. The definition of the ordering on $\Gamma_{B}$ gives $B s B \subseteq B t B$ iff $w(s) \geq w(t)$. We now verify the conditions of Definition 2.1. Condition (1) is clear. For (2), suppose $w(s) \geq w(t)$. Then $B s B \subseteq B t B$, and so $s+t \in B t B$, hence $B(s+t) B \subseteq B t B$. Therefore $w(s+t) \geq w(t)=\min \{w(s), w(t)\}$. To see (3), since additive notation is being used for values of $w$, the element $w(s)+w(t)$ refers to the image of $(B s B)(B t B)$ in $\Gamma_{B}$. As $s t \in(B s B)(B t B)$, Bst $B \subseteq(B s B)(B t B)$, hence $w(s t) \geq w(s)+w(t)$. For (4), it is clear that $\operatorname{im}(w)=w(s t(B))$, and if $\alpha \in \operatorname{st}(B), w\left(\alpha^{-1}\right)=-w(\alpha)$ since $w\left(\alpha^{-1}\right)+w(\alpha)$ corresponds to the ideal $\alpha^{-1} B \alpha B=\alpha^{-1} \alpha B=B$. Thus $\operatorname{st}(B) \subseteq \operatorname{st}(w)$, so $\operatorname{im}(w)=w(\operatorname{st}(w))$. Now to 
see $B=B_{w}$ and $J(B)=J_{w}, B s B \subseteq B$ (resp. $B s B=B$ ) iff $w(s) \geq w(1)=0$ (resp. $w(s)=0$ ). Since $B s B=B$ iff $s \in B-J(B)$, it follows that $B=\{s \in$ $S \mid w(s) \geq 0\}$ and $J(B)=\{s \in S \mid w(s)>0\}$.

Now, let $w$ be any value function on $S$ with $B=B_{w}$ and $J(B)=J_{w}$. For $b \in B^{*}, b^{-1} \in B$, so $w\left(b^{-1}\right) \geq 0$. But $0=w(1)=w\left(b b^{-1}\right) \geq w(b)+w\left(b^{-1}\right)$, so $0 \geq-w\left(b^{-1}\right) \geq w(b) \geq 0$, thus $w(b)=0$. If $s \in \operatorname{st}(w)$ with $w(s)=0$ then $w\left(s^{-1}\right)=0$, so $s, s^{-1} \in B$, hence $s \in B^{*}$. Therefore $B^{*}=\{s \in$ $s t(w) \mid w(s)=0\}$. If $s \in s t(w)$ then $w\left(s b s^{-1}\right)=w(b)$, so $w\left(s b s^{-1}\right) \geq 0$ iff $w(b) \geq 0$. Therefore $s B s^{-1}=B$, so $s \in s t(B)$. Conversely, if $s \in s t(B)$ then there is an $s_{0} \in s t(w)$ with $w(s)=w\left(s_{0}\right)$. Then $w\left(s s_{0}^{-1}\right)=0$ by Lemma 2.2, so $s s_{0}^{-1} \in(B-J(B)) \cap s t(B)=B^{*} \subseteq s t(w)$ by [ $\mathrm{W}_{2}$, Lemma 3.2]. So $s \in s t(w)$, hence $s t(w)=s t(B)$. Finally, by Lemma 2.2, $w: s t(B) \rightarrow i m(w)$ is a homomorphism. It is easy to see this map has kernel $B^{*}$ and is surjective, so $\Gamma_{B}=\operatorname{st}(B) / B^{*} \cong i m(w)$.

If $S$ is a central simple algebra and $B$ is a Dubrovin valuation ring of $S$, then $B$ has the extra property of Theorem 2.3 iff $B$ is integral over its center, by [ $\mathrm{M}_{2}$, Theorem 3.1 or $\mathrm{W}_{2}$, Theorem F]. The applications below and in [MW] deal exclusively with this finite dimensional situation.

An alternative approach to the above proof (and my original approach) in the case $S$ is a central simple algebra is the following. By [ $\mathrm{W}_{2}$, Theorem F] there is a Dubrovin valuation ring $B_{h}$ in $S \otimes_{F} F_{h}$ with $B_{h} \cap F_{h}=V_{h}$ and $B_{h} \cap S=B$, where $\left(F_{h}, V_{h}\right)$ is the Henselization of $(F, V)$. Since $F_{h}$ is Henselian, $B_{h} \cong M_{n}(R)$ where $R$ is the invariant valuation ring in the underlying division algebra $D$ of $S \otimes_{F} F_{h}$ (by Theorem 1.1 above). Defining $w$ on $S \otimes_{F} F_{h}$ (possibly modifying it to account for the isomorphism $B_{h} \cong M_{n}(R)$ ) as in the example after Definition 2.1 and restricting to $S$ gives the desired value function on $S$.

Frequently (as will be seen below and in [MW]) one wants to find a Dubrovin valuation ring in a central simple algebra and has a naturally occurring value function on the algebra. It would be useful to know which Dubrovin valuation rings come from (or give rise to) value functions. The following is a converse to Theorem 2.3 and was proved by A. Wadsworth.

Theorem 2.4. Let $w$ be a value function on $S$. Suppose $B_{w} / J_{w}$ is simple Artinian. Then $B_{w}$ is a Dubrovin valuation ring of $S$ with $J\left(B_{w}\right)=J_{w}$ and for all $s \in S-\{0\}$ there is an $\alpha \in \operatorname{st}\left(B_{w}\right)$ with $B_{w} s B_{w}=\alpha B_{w}$.

Proof. To show $B_{w}$ is a Dubrovin valuation ring of $S$ with $J\left(B_{w}\right)=J_{w}$, it suffices to show that if $s \in S-B_{w}$ then there are $b_{1}, b_{2} \in B_{w}$ with $s b_{1}$, $b_{2} s \in B_{w}-J_{w}$. Now since $s \notin B_{w}, w(s)<0$. There is a $t \in s t(w)$ with $w(s)=w(t)$. Hence $w\left(t^{-1}\right)=-w(t)>0$, so $t^{-1} \in B_{w}$. Then $w\left(s t^{-1}\right)=$ $w\left(t^{-1} s\right)=w(s)-w(t)=0$, so $s t^{-1}, t^{-1} s \in B_{w}-J_{w}$. Setting $b_{1}=b_{2}=t^{-1}$ shows that $B_{w}$ is a Dubrovin valuation ring. Let $s \in S-\{0\}$. Then as 
above there is a $t \in s t(w)$ with $w\left(s t^{-1}\right)=0$ hence $s t^{-1} \in B_{w}-J_{w}$. Thus $B_{w} s t^{-1} B_{w}=B_{w}$ as $J_{w}=J\left(B_{w}\right)$ is the unique maximal ideal of $B_{w}$. The same argument as in Theorem 2.3 shows $s t(w) \subseteq s t(B)$, so $B_{w} s B_{w}=t B_{w}$.

We combine Theorems 2.3 and 2.4 in the finite dimensional case for a useful corollary.

Corollary 2.5. Let $S$ be a central simple algebra and $B$ a Dubrovin valuation ring of $S$. Then there is a value function $w$ on $S$ with $B=B_{w}$ and $J(B)=J_{w}$ iff $B$ is integral over its center.

The final result of this section gives a number of properties of value functions on central simple algebras. In particular it relates a value function to the reduced norm $N r d$ on a central simple algebra $S$. For information on reduced norms, see [R, Chapter 2.9] or [J, §9]. Before proving the lemma we mention one property of $N r d$. Suppose $S$ is a central simple $F$-algebra, $V$ an integrally closed subring of $F$ and $B$ a subring of $S$ integral over $V$. If $b \in B$ then $N r d(b) \in V$. Furthermore, for $b \in B, b \in B^{*}$ iff $\operatorname{Nrd}(b) \in V^{*}$. To see this, let $f(x)$ be the reduced characteristic polynomial of $b$. As $b$ is integral over $V$ and $V$ is integrally closed, the minimal polynomial $p(x)$ of $b$ lies in $V[x]$, and since $f$ divides a power of $p, f$ lies in $V[x]$. The constant term of $f$ is up to sign equal to $N r d(b)$, so $N r d(b) \in V$. If $b \in B^{*}$ then $N r d\left(b^{-1}\right) \in V$, and $1=\operatorname{Nrd}(b) N r d\left(b^{-1}\right)$, so $N r d(b) \in V^{*}$. Conversely, if $N r d(b) \in V^{*}$, write $f(x)=\alpha_{0}+\alpha_{1} x+\cdots+\alpha_{n-1} x^{n-1}+x^{n}$ (so $\left.\alpha_{0}= \pm N r d(b)\right)$. Since $\alpha_{0} \in V^{*}$ we can write $1=b\left[-\alpha_{0}^{-1}\left(\alpha_{1}+\cdots+\alpha_{n-1} b^{n-2}+b^{n-1}\right)\right]$. This shows $b^{-1} \in B$, so $b \in B^{*}$.

Proposition 2.6. Let $S$ be a central simple $F$-algebra, $B$ a Dubrovin valuation ring of $S$ integral over $V=B \cap F$ and $w$ a value function on $S$ with $B=B_{w}$. Then

(1) Set $v=\left.w\right|_{F}$; $v$ is a valuation on $F$ corresponding to $V$.

(2) $s t(B)=\left\{s \in S^{*} \mid w(s)=\frac{1}{n} v(N r d(s))\right\}$, where $n=\sqrt{[S: F]}$.

(3) $w(s) \leq \frac{1}{n} v(N r d(s))$ in general.

(4) $w$ is uniquely determined on $S$ by $\left.w\right|_{F}$ and $B$.

Proof. (1) As $F^{*} \subseteq s t(B)=s t(w)$ by Theorem 2.3, $\left.w\right|_{F}=v$ is a valuation on $F$ by Lemma 2.2. Clearly the valuation ring of $v$ is $B \cap F$.

(2) For the inclusion $s t(B) \subseteq\left\{s \in S^{*} \mid w(s)=\frac{1}{n} v(N r d(s))\right\}$, if $s \in s t(B)$, then since $\left|\Gamma_{B}: \Gamma_{V}\right|$ divides $[S: F]=n^{2}, s^{n^{2}}=\alpha u$ with $\alpha \in F$ and $u \in B^{*}$. Then as $s \in s t(w), n^{2} w(s)=w\left(s^{n^{2}}\right)=w(\alpha)=v(\alpha)$, so $w(s)=$ $\frac{1}{n^{2}} v(\alpha)$. Also, $n^{2} v(N r d(s))=v\left(N r d\left(s^{n^{2}}\right)\right)=v(N r d(\alpha))+v(N r d(u))=n v(\alpha)$, thus $\frac{1}{n} v(N r d(s))=\frac{1}{n^{2}} v(\alpha)=w(s)$. For the reverse inclusion, take $s \in S^{*}$ with $w(s)=\frac{1}{n} v(N r d(s))$. There is an $s_{0} \in s t(w)$ with $w(s)=w\left(s_{0}\right)$. Since $w\left(s_{0}\right)=\frac{1}{n} v\left(N r d\left(s_{0}\right)\right)$, we have $v\left(N r d\left(s s_{0}^{-1}\right)\right)=0$, so $N r d\left(s s_{0}^{-1}\right) \in V^{*}$. Since 
$s s_{0}^{-1} \in B$ we see $s s_{0}^{-1} \in B^{*}$ by the above remark, so $s \in s_{0} B^{*} \subseteq s t(w)$. Thus $s t(B)=\left\{s \in S^{*} \mid w(s)=\frac{1}{n} v(N r d(s))\right\}$.

(3) Write $s=s_{0} u$ with $s_{0} \in s t(B)$ and $u \in B-J(B)$. Then

$$
\begin{aligned}
\frac{1}{n} v(N r d(s)) & =\frac{1}{n} v\left(N r d\left(s_{0} u\right)\right)=\frac{1}{n} v\left(N r d\left(s_{0}\right)\right)+\frac{1}{n} v(N r d(u)) \\
& \geq \frac{1}{n} v\left(\operatorname{Nrd}\left(s_{0}\right)\right)=w\left(s_{0}\right)=w(s)
\end{aligned}
$$

since $N r d(u) \in V$, hence $v(N r d(u)) \geq 0$.

(4) The equality $s t(B)=\left\{s \in S^{*} \mid w(s)=\frac{1}{n} v(N r d(s))\right\}$ shows that $w$ is uniquely determined on $s t(B)$ by $\left.w\right|_{F}=v$. If $s=s_{0} u$ with $s_{0} \in \operatorname{st}(B)$ and $u \in B-J(B)$, then $w(s)=w\left(s_{0}\right)$ by (4), so $w$ is uniquely determined an all of $S$.

\section{ApPLicAtions}

In this section we shall prove some results about integral Dubrovin valuation rings (that is, Dubrovin valuation rings integral over their center) by making heavy use of value functions. The similarity of value functions and valuations (in particular property (1) of Lemma 2.2) allow results about invariant valuation rings to be generalized to integral Dubrovin valuation rings. The style of argument used in [MW] is similar to the arguments below. By combining the Henselization theorem [ $\mathrm{W}_{2}$, Theorem B] with results about integral Dubrovin valuation rings we are able to obtain results about arbitrary Dubrovin valuation rings (see for instance Corollary 3.6).

Lemma 3.1. Suppose $B$ is an integral Dubrovin valuation ring of a central simple algebra $S, F$ a subfield of $Z(S), V=B \cap F$, and $w$ is a value function on $S$ corresponding to $B$ with $\left.w\right|_{F}=v$. Take $a_{1}, \ldots, a_{e} \in \operatorname{st}(B)$ such that the $w\left(a_{i}\right)$ are distinct $\bmod \Gamma_{V}$, and $u_{1}, \ldots, u_{f} \in B-J(B)$ such that $\overline{u_{1}}, \ldots, \overline{u_{f}}$ are linearly independent over $\bar{V}$. Then the elements $\left\{a_{i} u_{j} \mid 1 \leq i \leq e, 1 \leq\right.$ $j \leq f\}$ are linearly independent over $F$. Furthermore, if $x=\sum_{i, j} \alpha_{i j} a_{i} u_{j}$ with $\alpha_{i j} \in F$, then $w(x)=\min _{i, j}\left\{v\left(\alpha_{i j}\right)+w\left(a_{i}\right)\right\}$.

Proof. Suppose $\alpha_{i j} \in F$. For a fixed $i$ there is a $j_{0}$ with $v\left(\alpha_{i j_{0}}\right) \leq v\left(\alpha_{i j}\right)$ for all $j$. Then $\alpha_{i j_{0}}^{-1} \alpha_{i j} \in V$ and $\overline{\sum_{j} \alpha_{i j_{0}}^{-1} \alpha_{i j} u_{j}}=\sum_{j} \overline{\alpha_{i j_{0}}^{-1} \alpha_{i j}} \overline{u_{j}} \neq \overline{0}$ by the $\bar{V}$ independence of the $\overline{u_{j}}$. Therefore $\sum_{j} \alpha_{i j_{0}}^{-1} \alpha_{i j} u_{j} \in B-J(B)$, so $w\left(\sum_{j} \alpha_{i j} u_{j}\right)=$ $v\left(\alpha_{i j_{0}}\right)=\min _{j}\left\{v\left(\alpha_{i j}\right)\right\} \in \Gamma_{V}$. Now $w\left(\sum_{j} \alpha_{i j} a_{i} u_{j}\right)=w\left(a_{i}\right)+w\left(\sum_{j} \alpha_{i j} u_{j}\right)=$ $w\left(a_{i}\right)+\min _{j}\left\{v\left(\alpha_{i j}\right)\right\}$, since the $a_{i} \in \operatorname{st}(B)$. As the $w\left(a_{i}\right)$ are distinct mod $\Gamma_{V}$, the elements $\min _{j}\left\{v\left(\alpha_{i j}\right)+w\left(a_{i}\right)\right\}$ are distinct, so $w\left(\sum_{i, j} \alpha_{i j} a_{i} u_{j}\right)=$ $\min _{i, j}\left\{v\left(\alpha_{i j}\right)+w\left(a_{i}\right)\right\}$, by Lemma 2.2. This implies that if $\sum_{i, j} \alpha_{i j} a_{i} u_{j}=0$ then all $\alpha_{i j}=0$, so the $a_{i} u_{j}$ are linearly independent over $F$.

Given $B$ a Dubrovin valuation ring of $S$ lying over $V$, let $\varepsilon=\varepsilon_{B / V}$ be the number of elements in the set $\left\{\gamma \in \Gamma_{B} \mid 0 \leq \gamma<\alpha \forall \alpha \in \Gamma_{V}, \alpha>0\right\}$. It is 
easy to see that if $0=\gamma_{1}<\cdots<\gamma_{\varepsilon}<\Gamma_{V}^{+}=\left\{\alpha \in \Gamma_{V} \mid \alpha>0\right\}$ then the $\gamma_{i}$ are distinct $\bmod \Gamma_{V}$, so $\varepsilon_{B / V} \leq e_{B / V}$. It is well known (cf. [B, pp. 424, 427 or $\mathrm{E}, 13.5,18.6])$ that if $K$ is a finite field extension of $F$ and $B$ is a valuation ring of $K$ (lying over $V$ ), then $B$ is a finite $V$-module iff $\varepsilon_{B / V} f_{B / V}=[K: F]$. This can be generalized to Dubrovin valuation rings:

Proposition 3.2. Suppose $B$ is a Dubrovin valuation ring of a central simple algebra $S$ with $F=Z(S)$ and $V=B \cap F$. Then $B$ is a finite $V$-module iff $\varepsilon_{B / V} f_{B / V}=[S: F]$. When this occurs, $B$ is integral and defectless over $V$.

Proof. Suppose $\varepsilon_{B, V} f_{B, V}=[S: F]$. Since $\varepsilon_{B, V} \leq e_{B, V}, e_{B, V} f_{B, V}=$ $[S: F]$. Then $B$ is integral and defectless by [ $\mathrm{W}_{2}$, Theorems $C$ and $\left.F\right]$. Let $w$ be a value function on $S$ corresponding to $B$. Choose $a_{1}, \ldots, a_{\varepsilon} \in \operatorname{st}(B)$ such that $\left\{w\left(a_{i}\right) \mid 1 \leq i \leq \varepsilon\right\}$ represents all the elements $\gamma$ with $0 \leq \gamma<\Gamma_{V}^{+}$, and $u_{1}, \ldots, u_{f} \in B-J(B)$ such that $\overline{u_{1}}, \ldots, \overline{u_{f}}$ form a $\bar{V}$-basis of $\bar{B}$. Since the $w\left(a_{i}\right)$ are distinct mod $\Gamma_{V}$, Lemma 3.1 shows that the elements $a_{i} u_{j}$ are linearly independent over $F$, hence form an $F$-basis of $S$ by the defectlessness of $B / V$. Take $x=\sum_{i, j} \alpha_{i j} a_{i} u_{j} \in B, \alpha_{i j} \in F$. Then $w(x)=$ $\min _{i, j}\left\{v\left(\alpha_{i j}\right)+w\left(a_{i}\right)\right\} \geq 0$, so for all $i, j, v\left(\alpha_{i j}\right)+w\left(a_{i}\right) \geq 0$. If $v\left(\alpha_{i j}\right)<0$ for some $i, j$ then $0<-v\left(\alpha_{i j}\right) \leq w\left(a_{i}\right)$ which would contradict the choice of the $a_{i}$. Therefore all $\alpha_{i j} \in V$, so $B \subseteq \sum_{i, j} V a_{i} u_{j}$. Since all $a_{i} u_{j} \in B$, $B=\sum_{i, j} V a_{i} u_{j}$, so $B$ is a finite $V$-module.

Conversely, suppose $B$ is a finite $V$-module. Since $B$ is a torsion free $V$-module, $B$ is free (as $V$ is a valuation ring), and since $B \otimes_{V} F \cong S$, $\operatorname{rank}_{V}(B)=[S: F]$. Let $s_{1}, \ldots, s_{\varepsilon} \in \operatorname{st}(B)$ be such that $\left\{s_{1} B^{*}, \ldots, s_{\varepsilon} B^{*}\right\}=$ $\left\{\gamma \in \Gamma_{B} \mid 0 \leq \gamma<\alpha \forall \alpha \in \Gamma_{B}^{+}\right\}$and $B=s_{1} B \supset s_{2} B \supset \cdots \supset s_{\varepsilon} B \supset J(V) B$. Set $M_{i}=s_{i} B$ and $M_{\varepsilon+1}=J(V) B$. By definition of the $s_{i}, M_{i} J(B) \subseteq M_{i+1}$, so the $M_{i} / M_{i+1}$ are (right) $\bar{B}$-modules. $M_{i} / M_{i+1}$ is free as a $\bar{B}$-module of rank 1 . To see this, clearly $\overline{s_{i}}=s_{i}+M_{i+1}$ generates $M_{i} / M_{i+1}$. If $\overline{s_{i}} \bar{b}=\overline{0}$ for $b \in B, s_{i} b \in M_{i+1}$, so $s_{i} b \in s_{i+1} B$. If $b \in B-J(B)$, we then have $B s_{i} b B=s_{i} B b B=s_{i} B \nsubseteq s_{i+1} B$, so $b \in J(B)$, hence $\bar{b}=\overline{0}$. Therefore we get

$$
\begin{aligned}
{[S: F] } & =\operatorname{rank}_{V}(B)=\operatorname{rank}_{\bar{V}}(B / J(V) B)=\sum_{i=1}^{\varepsilon} \operatorname{rank}_{\bar{V}}\left(M_{i} / M_{i+1}\right) \\
& =\sum_{i=1}^{\varepsilon}[\bar{B}: \bar{V}] \operatorname{rank}_{\bar{B}}\left(M_{i} / M_{i+1}\right)=\sum_{i=1}^{\varepsilon} f_{B / V}=\varepsilon_{B / V} f_{B / V} .
\end{aligned}
$$

Therefore $\varepsilon_{B / V} f_{B / V}=[S: F]$.

In dealing with central simple algebras one frequently has to consider tensor products of algebras and extension of scalars. When studying valuation theory it is very useful to be able to determine properties of Dubrovin valuation rings in a tensor product given information about each of the pieces. For invariant valuation rings, Theorem 1 of $\left[M_{1}\right]$ has proven to be quite useful, as seen in 
[JW $\left.{ }_{2}\right]$. In that result a function was defined and shown to be a valuation. The proof was quite long due to the difficulty in proving the multiplicative property $v(a b)=v(a)+v(b)$. The inequality $v(a b) \geq v(a)+v(b)$ was quite easy to show however. The following is a generalization to Dubrovin valuation rings of that result, and the idea of the proof is much the same, but is much easier due to the less restrictive multiplicative property of value functions on central simple algebras. Using the terminology of [ $\left.\mathrm{W}_{2}\right]$, if $S \subseteq T$ are central simple algebras with $B \subseteq S$ and $A \subseteq T$ Dubrovin valuation rings such that $A \cap S=B$, $J(B) \subseteq J(A)$ and $s t(B) \subseteq s t(A)$, then we say $A$ is a compatible extension of $B$. Note that for a compatible extension $A / B$ there are natural inclusions $\Gamma_{B} \hookrightarrow \Gamma_{A}$ and $\bar{B} \hookrightarrow \bar{A}$.

Theorem 3.3. Let $S_{1}$ and $S_{2}$ be central simple algebras, $F \subseteq Z\left(S_{i}\right), B_{i} \subseteq S_{i}$ integral Dubrovin valuation rings with $B_{1} \cap F=B_{2} \cap F=V$. Suppose

(1) $\Gamma_{B_{1}} \cap \Gamma_{B_{2}}=\Gamma_{V}$,

(2) $\overline{B_{1}} \otimes_{\bar{V}} \overline{B_{2}}$ is simple Artinian,

(3) $B_{1} / V$ is defectless.

If $B$ is a Dubrovin valuation ring in $S=S_{1} \otimes_{F} S_{2}$ then $B$ is integral, $\Gamma_{B}=$ $\Gamma_{B_{1}}+\Gamma_{B_{2}}, \bar{B}=\overline{B_{1}} \otimes_{\bar{V}} \overline{B_{2}}, \delta(B)=\delta\left(B_{2}\right)$ and the matrix size of $S_{1} \otimes_{F} S_{2}$ divides the matrix size of $\overline{B_{1}} \otimes_{\bar{V}} \overline{B_{2}}$. Furthermore, $B$ can be chosen to be a compatible extension of $B_{1}$ and $B_{2}$.

Proof. Since $\left|\Gamma_{B_{1}}: \Gamma_{V}\right|<\infty$ (by (3)), there is a unique order preserving homomorphism $\Gamma_{B_{1}} \hookrightarrow \Gamma_{V} \otimes_{\mathbf{Z}} \mathbf{Q} \hookrightarrow \Gamma_{B_{2}} \otimes_{\mathbf{Z}} \mathbf{Q}=\Delta_{B_{2}}, \Delta_{B_{2}}$ the divisible hull of $\Gamma_{B_{2}}$. The intersection $\Gamma_{B_{1}} \cap \Gamma_{B_{2}}$ is then computed in $\Delta_{B_{2}}$. Let $a_{1}, \ldots, a_{e} \in \operatorname{st}(B)$ be representatives of $\Gamma_{B_{1}} / \Gamma_{V}$ and $u_{1}, \ldots, u_{f} \in B-J(B)$ map to a $\bar{V}$-basis of $\overline{B_{1}}$ with $a_{1}=u_{1}=1$. Since $B_{1} / V$ is defectless the $a_{i} u_{j}$ are an $F$-basis of $S_{1}$ by Lemma 3.1. Thus every element in $S$ can be written uniquely in the form $\sum_{i, j} a_{i} u_{j} \otimes s_{i j}$. Let $w_{i}$ be a value function on $S_{i}$ corresponding to $B_{i}$ with $\left.w_{1}\right|_{F}=\left.w_{2}\right|_{F}=v$. Define $w$ on $S$ by

$$
w\left(\sum_{i, j} a_{i} u_{j} \otimes s_{i j}\right)=\min _{i, j}\left\{w_{1}\left(a_{i}\right)+w_{2}\left(s_{i j}\right)\right\} .
$$

Let $B=\{s \in S \mid w(s) \geq 0\}$ and $J=\{s \in S \mid w(s)>0\}$. We will show that $w$ is a value function on $S$ and $B / J$ is simple Artinian, so by Theorem 2.4, $B$ will be an integral Dubrovin valuation ring of $S$. We first show that

(a) $w(s+t) \geq \min \{w(s), w(t)\}$,

(b) $w(s t) \geq w(s)+w(t)$,

(c) $w\left(s_{1} \otimes s_{2}\right)=w_{1}\left(s_{1}\right)+w_{2}\left(s_{2}\right)$.

Property (c) shows that $B_{1} \otimes_{V} B_{2} \subseteq B$ and $J\left(B_{i}\right)=B_{i} \cap J$, hence $\overline{B_{1}}, \overline{B_{2}} \hookrightarrow$ $B / J$. To demonstrate these properties, let

$$
s=\sum_{i, j} a_{i} u_{j} \otimes s_{i j} \quad \text { and } \quad t=\sum_{i, j} a_{i} u_{j} \otimes t_{i j} .
$$


(a) $w(s+t)=\min _{i, j}\left\{w_{1}\left(a_{i}\right)+w_{2}\left(s_{i j}+t_{i j}\right)\right\}$. Suppose the minimum occurs at $i, j=k, l$, and that $w_{2}\left(s_{k l}\right) \leq w_{2}\left(t_{k l}\right)$. Then

$$
\begin{aligned}
w(s+t) & =w_{1}\left(a_{k}\right)+w_{2}\left(s_{k l}+t_{k l}\right) \geq w_{1}\left(a_{k}\right)+w_{2}\left(s_{k l}\right) \geq \min _{i, j}\left\{w_{1}\left(a_{i}\right)+w_{2}\left(s_{i j}\right)\right\} \\
& =w(s) \geq \min \{w(s), w(t)\} .
\end{aligned}
$$

(c) Say $s_{1}=\sum_{i, j} \alpha_{i j} a_{i} u_{j}$ with $\alpha_{i j} \in F$. Then $s_{1} \otimes s_{2}=\sum_{i, j} a_{i} u_{j} \otimes \alpha_{i j} s_{2}$, so $w\left(s_{1} \otimes s_{2}\right)=\min _{i, j}\left\{w_{1}\left(a_{i}\right)+w_{2}\left(\alpha_{i j} s_{2}\right)\right\}$

$$
\begin{aligned}
& =\min _{i, j}\left\{w_{1}\left(a_{i}\right)+v\left(\alpha_{i j}\right)+w_{2}\left(s_{2}\right)\right\}=\min _{i, j}\left\{w_{1}\left(a_{i}\right)+v\left(\alpha_{i j}\right)\right\}+w_{2}\left(s_{2}\right) \\
& =w_{1}\left(s_{1}\right)+w_{2}\left(s_{2}\right) .
\end{aligned}
$$

(b) Let $\sigma_{i j}=a_{i} u_{j} \otimes s_{i j}$ and $\tau_{i j}=a_{i} u_{j} \otimes t_{i j}$. So $s t=\sum_{i, j, k, l} \sigma_{i j} \tau_{k l}$. Then by (a) and (c),

$$
w(s t) \geq \min _{i, j, k, l}\left\{w\left(\sigma_{i j} \tau_{k l}\right)\right\} \geq \min _{i, j, k, l}\left\{w\left(\sigma_{i j}\right)+w\left(\tau_{k l}\right)\right\}=w(s)+w(t) .
$$

Since $\overline{B_{i}} \hookrightarrow B / J$ and their images commute there is a $\bar{V}$-algebra homomorphism $\overline{B_{1}} \otimes_{\bar{V}} \overline{B_{2}} \rightarrow B / J$ given by $\overline{b_{1}} \otimes \overline{b_{2}} \mapsto b_{1} \otimes b_{2}+J$. This map is injective by the simplicity of $\overline{B_{1}} \otimes_{\bar{V}} \overline{B_{2}}$. For surjectivity, let $b=\sum_{i, j} a_{i} u_{j} \otimes s_{i j} \in B-J$. So $w(b)=0$, hence some $w_{1}\left(a_{i}\right)+w_{2}\left(s_{i j}\right)=0$. Since $\Gamma_{B_{1}} \cap \Gamma_{B_{2}}=\Gamma_{V}$ this can only happen for $i=1$ as $a_{1}=1$, and so all $w_{2}\left(s_{1 j}\right) \geq 0$ and $w_{2}\left(s_{1 j}\right)=0$ for some $j$. As the elements $\sum_{j} a_{i} u_{j} \otimes s_{i j}$ have distinct $w$-values, $b+J=\left(\sum_{j} u_{j} \otimes s_{1 j}\right)+J$. Thus $\sum_{j} \overline{u_{j}} \otimes \overline{s_{1 j}} \rightarrow b+J$. Hence $B / J \cong \overline{B_{1}} \otimes_{\bar{V}} \overline{B_{2}}$, so is simple Artinian.

By $(\mathrm{c}), \operatorname{st}\left(w_{1}\right) \otimes s t\left(w_{2}\right) \subseteq s t(w)$ and it is clear from the definition of $w$ that $\operatorname{im}(w)=w\left(s t\left(w_{1}\right) \otimes s t\left(w_{2}\right)\right)$. Hence the hypotheses of Theorem 2.4 are satisfied. So $B$ is an integral Dubrovin valuation ring with $w$ a value function on $S$ corresponding to $B$. The definition of $w$ shows $\Gamma_{B}=\operatorname{im}(w)=\Gamma_{B_{1}}+\Gamma_{B_{2}}$ and the above argument gives $\bar{B}=\overline{B_{1}} \otimes_{\bar{V}} \overline{B_{2}}$. Since $J(B) \cap B_{i}=J\left(B_{i}\right)$ and $s t\left(B_{i}\right) \subseteq s t(B), B / B_{i}$ is a compatible extension. The matrix size of $S$ divides that of $n_{B}=t_{B}$, which is the matrix size of $\bar{B}=\overline{B_{1}} \otimes_{\bar{V}} \overline{B_{2}}$ since $B$ is integral. Finally to show $\delta(B)=\delta\left(B_{2}\right)$, by [W $\mathrm{W}_{2}$, Theorem C],

$$
\begin{aligned}
{[S: Z(S)] } & =\left|\Gamma_{B}: \Gamma_{Z(B)}\right|[\bar{B}: \overline{Z(B)}] \delta(B), \\
{\left[S_{1}: Z\left(S_{1}\right)\right] } & =\left|\Gamma_{B_{1}}: \Gamma_{Z\left(B_{1}\right)}\right|\left[\overline{B_{1}}: \overline{Z\left(B_{1}\right)}\right], \\
{\left[S_{2}: Z\left(S_{2}\right)\right] } & =\left|\Gamma_{B_{2}}: \Gamma_{Z\left(B_{2}\right)}\right|\left[\overline{B_{2}}: \overline{Z\left(B_{2}\right)}\right] \delta\left(B_{2}\right) .
\end{aligned}
$$

Since $S=S_{1} \otimes_{F} S_{2}$ and $Z(S)=Z\left(S_{1}\right) \otimes_{F} Z\left(S_{2}\right)$,

$$
[S: Z(S)]=\left[S_{1}: Z\left(S_{1}\right)\right]\left[S_{2}: Z\left(S_{2}\right)\right] \text {. }
$$

Replacing $S, S_{1}$ and $S_{2}$ by $Z(S), Z\left(S_{1}\right)$ and $Z\left(S_{2}\right)$ respectively and applying what has been proven, we see that $\Gamma_{Z(B)}=\Gamma_{Z\left(B_{1}\right)}+\Gamma_{Z\left(B_{2}\right)}$ and $\overline{Z(B)}=$ $\overline{Z\left(B_{1}\right)} \otimes_{\bar{V}} \overline{Z\left(B_{2}\right)}$. This residue property along with $\bar{B}=\overline{B_{1}} \otimes_{\bar{V}} \overline{B_{2}}$ yields

$$
[\bar{B}: \overline{Z(B)}]=\left[\overline{B_{1}}: \overline{Z\left(B_{1}\right)}\right]\left[\overline{B_{2}}: \overline{Z\left(B_{2}\right)}\right] \text {. }
$$


We note that $\Gamma_{B}=\Gamma_{B_{1}}+\Gamma_{B_{2}}$ and $\Gamma_{Z(B)}=\Gamma_{Z\left(B_{1}\right)}+\Gamma_{Z\left(B_{2}\right)}$ together with $\Gamma_{B_{1}} \cap$ $\Gamma_{B_{2}}=\Gamma_{V}$ implies the isomorphism $\Gamma_{B} / \Gamma_{Z(B)} \cong \Gamma_{B_{1}} / \Gamma_{Z\left(B_{1}\right)} \oplus \Gamma_{B_{2}} / \Gamma_{Z\left(B_{2}\right)}$. This then gives

$$
\left|\Gamma_{B}: \Gamma_{Z(B)}\right|=\left|\Gamma_{B_{1}}: \Gamma_{Z\left(B_{1}\right)}\right|\left|\Gamma_{B_{2}}: \Gamma_{Z\left(B_{2}\right)}\right| .
$$

Putting all this together gives $\delta(B)=\delta\left(B_{2}\right)$. Since all Dubrovin valuation rings in $S$ extending $V$ are conjugate by [ $\mathrm{W}_{2}$, Theorem A], the theorem is proven.

There are a number of occasions when the tensor product of Dubrovin valuation rings is again a Dubrovin valuation ring, as seen in [ $\left.\mathrm{W}_{2}, \S 3\right]$. As seen in the above proof $B_{1} \otimes_{V} B_{2} \subseteq B$. The next result gives an easily checked condition on when $B_{1} \otimes_{V} B_{2}$ is a Dubrovin valuation ring in $S_{1} \otimes_{F} S_{2}$.

Theorem 3.4. With the notation of Theorem 3.3, $B_{1} \otimes_{V} B_{2}$ is a Dubrovin valuation ring iff any nonnegative element of $\Gamma_{B_{1}}+\Gamma_{B_{2}}$ can be written in the form $\gamma_{1}+\gamma_{2}$ with $\gamma_{i} \in \Gamma_{B_{i}}$ and $\gamma_{1}, \gamma_{2} \geq 0$.

Proof. Let $B$ be as in the proof of Theorem 3.3. Since $B_{1} \otimes_{V} B_{2} \subseteq B$ it follows from $\left[\mathrm{D}_{2}, \S 2\right.$, Theorem 1] that $B_{1} \otimes_{V} B_{2}$ is a Dubrovin valuation ring iff $B_{1} \otimes_{V} B_{2}=B$. For an ordered group $\Gamma$, set $\Gamma^{\bullet}=\{\gamma \in \Gamma \mid \gamma \geq$ $0\}=\Gamma^{+} \cup\{0\}$. The condition above can then be stated as $\Gamma_{B}^{\circ}=\Gamma_{B_{1}}^{\circ}+\Gamma_{B_{2}}^{\circ}$. Suppose $\Gamma_{B}^{\circ}=\Gamma_{B_{1}}^{\circ}+\Gamma_{B_{2}}^{\circ}$. Take $b=\sum_{i, j} a_{i} u_{j} \otimes s_{i j} \in B$. Then $w(b) \geq 0$, so for all $i, j, w_{1}\left(a_{i}\right)+w_{2}\left(s_{i j}\right) \geq 0$. Therefore there are $\alpha_{i j} \in \operatorname{st}\left(B_{1}\right) \cap B_{1}$, $\beta_{i j} \in \operatorname{st}\left(B_{2}\right) \cap B_{2}$ with $w_{1}\left(a_{i}\right)+w_{2}\left(s_{i j}\right)=w_{1}\left(\alpha_{i j}\right)+w_{2}\left(\beta_{i j}\right)$. So $w_{1}\left(a_{i}\right)-$ $w_{1}\left(\alpha_{i j}\right)=w_{2}\left(\beta_{i j}\right)-w_{2}\left(s_{i j}\right) \in \Gamma_{B_{1}} \cap \Gamma_{B_{2}}=\Gamma_{V}$. So there are $g_{i j} \in B_{1}-J\left(B_{1}\right)$, $h_{i j} \in B_{2}-J\left(B_{2}\right), x_{i j} \in F^{*}$ with $a_{i}=\alpha_{i j} g_{i j} x_{i j}, s_{i j}=\beta_{i j} h_{i j} x_{i j}^{-1}$. Hence $a_{i} u_{j} \otimes s_{i j}=\alpha_{i j} g_{i j} u_{j} \otimes \beta_{i j} h_{i j} \in B_{1} \otimes_{V} B_{2}$. So $b \in B_{1} \otimes_{V} B_{2}$, hence $B=B_{1} \otimes_{V} B_{2}$.

Conversely, suppose $B=B_{1} \otimes_{V} B_{2}$. Take $b \in B=B_{1} \otimes_{V} B_{2}$. We will show that $w(b) \in \Gamma_{B_{1}}+\Gamma_{B_{2}}$. Write $b=\sum_{i} d_{i} \otimes e_{i}$ with $d_{i} \in B_{1}, e_{i} \in B_{2}$, and say $d_{i}=\sum_{s, t} \alpha_{s t i} a_{s} u_{t}$. Since $d_{i} \in B_{1}, w_{1}\left(d_{i}\right) \geq 0$, so $w_{1}\left(\alpha_{s t i} a_{s}\right) \geq 0$ for all $s, t, i$. Also, $b=\sum_{s, t} a_{s} u_{t} \otimes\left(\sum_{i} \alpha_{s t i} e_{i}\right)$. Pick $s_{0}, t_{0}$ with $w(b)=$ $w_{1}\left(a_{s_{0}}\right)+w_{2}\left(\sum_{i} \alpha_{s_{0} t_{0}} e_{i}\right)$ and $i_{0}$ with $v\left(\alpha_{s_{0} t_{0} i_{0}}\right)=\min _{i}\left\{v\left(\alpha_{s_{0} t_{0} i}\right)\right\}$. Therefore $\alpha_{s_{0} t_{0} i_{0}}^{-1} \alpha_{s_{0} t_{0} i} \in V$, so $e=\sum_{i} \alpha_{s_{0} t_{0} i_{0}}^{-1} \alpha_{s_{0} t_{0} i} e_{i} \in B_{2}$. Hence $w(b)=w_{1}\left(\alpha_{s_{0} t_{0} i_{0}} a_{s_{0}}\right)+$ $w_{2}(e) \in \Gamma_{B_{1}}^{\circ}+\Gamma_{B_{2}}^{\bullet}$. Therefore $w(B) \subseteq \Gamma_{B_{1}}^{\circ}+\Gamma_{B_{2}}^{\circ}$. Since $w(B)=\Gamma_{B}^{\circ} \supseteq \Gamma_{B_{1}}^{\circ}+\Gamma_{B_{2}}^{\circ}$, we get $\Gamma_{B}^{\circ}=\Gamma_{B_{1}}^{\circ}+\Gamma_{B_{2}}^{\circ}$.

As an easy corollary of Theorem 3.3 we get $\left[\mathrm{M}_{1}\right.$, Theorem 1].

Corollary 3.5. With the notation above, suppose along with the hypotheses of Theorem 3.3 that $S_{1}$ and $S_{2}$ are division rings, $B_{1}$ and $B_{2}$ are invariant valuation rings and that $\overline{B_{1}} \otimes_{\bar{V}} \overline{B_{2}}$ is a division ring. Then $S_{1} \otimes_{F} S_{2}$ is a division ring and $B$ is an invariant valuation ring.

Proof. Since the matrix size of $S_{1} \otimes_{F} S_{2}$ divides the matrix size of $\overline{B_{1}} \otimes_{\bar{V}}$ $\overline{B_{2}}, S_{1} \otimes_{F} S_{2}$ is a division ring. Since $\bar{B}$ is a division ring, $n_{B}=t_{B}=1$ 
by $\left[\mathrm{W}_{2}\right.$, Theorem $\left.\mathrm{F}\right]$ as $B$ is integral. Thus $\left[\mathrm{M}_{1}\right.$, Theorem 2] shows that $B$ is an invariant valuation ring.

The added generality of the residue condition (2) in Theorem 3.3 yields new information about division algebras over Henselian fields (beyond that given by Corollary 3.5). If $(F, V)$ is a Henselian field, $D_{1}, D_{2} F$-central division algebras with $V_{D_{i}} \subset D_{i}$ the invariant valuation rings lying over $V$ and satisfying the hypotheses of Theorem 3.3, then if $D$ is the underlying division algebra of $D_{1} \otimes_{F} D_{2}$, then $\Gamma_{V_{D}}=\Gamma_{V_{D_{1}}}+\Gamma_{V_{D_{2}}}$ and $\overline{V_{D}} \sim \overline{V_{D_{1}}} \otimes_{\bar{V}} \overline{V_{D_{2}}}$. Furthermore it is not hard to see that the matrix size of $D_{1} \otimes_{F} D_{2}$ is equal to the matrix size of $\overline{V_{D_{1}}} \otimes \bar{V} \overline{V_{D_{2}}}$.

We can combine Theorem 3.3 with $\left[\mathrm{W}_{2}\right.$, Theorem $\mathrm{B}$ ] to get information about arbitrary Dubrovin valuation rings. For two central simple $F$-algebras $C$ and $C^{\prime}$, we write $C \sim C^{\prime}$ when $[C]=\left[C^{\prime}\right]$ in $\operatorname{Br}(F)$, that is when the underlying division algebras of $C$ and $C^{\prime}$ are $F$-isomorphic.

Corollary 3.6. Suppose $S_{1}$ and $S_{2}$ are central simple $F$-algebras, $B_{i} \subseteq S_{i}$ Dubrovin valuation rings lying over $V$ with $\Gamma_{B_{1}} \cap \Gamma_{B_{2}}=\Gamma_{V}, \overline{B_{1}} \otimes_{\bar{V}} \overline{B_{2}}$ simple Artinian and $B_{1} / V$ defectless. If $B$ is a Dubrovin valuation ring in $S=S_{1} \otimes_{F} S_{2}$ lying over $V$ then $\Gamma_{B}=\Gamma_{B_{1}}+\Gamma_{B_{2}}$ and $\bar{B} \sim \overline{B_{1}} \otimes_{\bar{V}} \overline{B_{2}}$.

Proof. Let $\left(F_{h}, V_{h}\right)$ be the Henselization of $(F, V)$. Say $S_{i} \otimes_{F} F_{h}=M_{n_{i}}\left(D_{i}\right)$ and $S \otimes_{F} F_{h}=M_{n}(D)$ for $D_{1}, D_{2}, D$ division algebras. Since $F_{h}$ is Henselian there are invariant valuation rings $R_{1}, R_{2}$ and $R$ in $D_{1}, D_{2}$ and $D$ respectively lying over $V_{h}$. By [W ${ }_{2}$, Theorem B] we have $\Gamma_{B_{i}}=\Gamma_{R_{i}}, \Gamma_{B}=\Gamma_{R}$, $\overline{B_{i}} \sim \overline{R_{i}}$ and $\bar{B} \sim \bar{R}$. Thus $\Gamma_{R_{1}} \cap \Gamma_{R_{2}}=\Gamma_{V_{h}}, \overline{R_{1}} \otimes \overline{V_{h}} \overline{R_{2}}$ is simple Artinian and $R_{1} / V$ is defectless, since $\delta\left(R_{1}\right)=\delta\left(B_{1}\right)=1$ by [W ${ }_{2}$, Theorem C]. So by Theorem 3.3, if $B^{\prime}$ is a Dubrovin valuation ring in $D_{1} \otimes_{F} D_{2}, \Gamma_{B^{\prime}}=\Gamma_{R_{1}}+\Gamma_{R_{2}}$ and $\overline{B^{\prime}}=\overline{R_{1}} \otimes_{\overline{V_{h}}} \overline{R_{2}}$. Since $S \otimes_{F} F_{h} \sim D_{1} \otimes_{F} D_{2}$, if $B_{h}$ is a Dubrovin valuation ring in $S \otimes_{F} F_{h}$, then $\Gamma_{B_{h}}=\Gamma_{B^{\prime}}$ and $\overline{B_{h}} \sim \overline{B^{\prime}}$ by $\left[\mathrm{W}_{2}\right.$, Corollary 3.5]. So applying $\left[\mathrm{W}_{2}\right.$, Theorem $\left.\mathrm{B}\right]$ again gives the result.

\section{EXAMPLES}

The situation is more complicated for nonintegral Dubrovin valuation rings. First of all one does not have a value function to use. Secondly, the nonintegrality of a Dubrovin valuation ring $B$ means that there is an overring $A$ such that the valuation ring $\widetilde{V}=V /(J(A) \cap V)$ in $\overline{Z(A)}$ extends in more than one way to $Z(\bar{A})\left[\mathrm{W}_{2}\right.$, Theorem $\left.\mathrm{F}\right]$. This gives room for complications to occur, as illustrated in the following examples. We first prove a proposition that will help us to construct these examples. Recall that an invariant valuation ring $R$ of an $F$-central division algebra $D$ is called inertial if $[\bar{R}: \bar{V}]=[D: F]$ and $Z(\bar{R})=\bar{V}$, where $V=R \cap F$. It then follows that $\Gamma_{R}=\Gamma_{V}$ and that $R / V$ is defectless. 
Proposition 4.1. Let $S$ be a central simple $F$-algebra and $V$ a valuation ring of $F$. Suppose $A$ is an Azumaya algebra over $V$ with quotient ring $S$ and $\bar{A}=A / J(V) A$ a division ring. Then $S$ is a division ring and $A$ is an inertial invariant valuation ring of $S$.

Proof. First assume $F$ is Henselian, and say $S=M_{n}(D)$, where $D$ is a division ring. By [JW ${ }_{2}$, Proposition 2.5] $A \cong M_{n}\left(V_{D}\right)$, where $V_{D}$ is the invariant valuation ring of $D$ lying over $V$. Therefore $\bar{A} \cong M_{n}\left(\overline{V_{D}}\right)$, hence $n=1$, so $S=D$ is a division ring and $A$ is an invariant valuation ring. $A$ is inertial also by [ $\mathrm{JW}_{2}$, Proposition 2.5]. In general, let $\left(F_{h}, V_{h}\right)$ be the Henselization of $(F, V)$, and set $S_{h}=S \otimes_{F} F_{h}$ and $A_{h}=A \otimes_{V} V_{h}$. Then $A_{h}$ is Azumaya over $V_{h}$ and $\overline{A_{h}}=\bar{A}$. So by the above argument $A_{h}$ is an inertial valuation ring in the division algebra $S_{h}$. Therefore by [ $\mathrm{M}_{1}$, Theorem 2], $S$ is a division algebra and $A=A_{h} \cap S$ is an inertial valuation ring.

This proposition will be used as follows: Let $S$ be the quaternion algebra $\left(\frac{a, b}{F}\right)$ for $a, b \in F^{*}$. This is the $F$-algebra with generators $i, j$ and relations $i^{2}=a, j^{2}=b$ and $i j=-j i$ (see [L, Chapter 3] for more on quaternion algebras). Suppose that $a, b \in V^{*}$ and $\left(\frac{\bar{a}, \bar{b}}{\bar{V}}\right)$ is a division ring. The subring $A=\left(\frac{a, b}{V}\right)$ of $S$ is an Azumaya algebra over $V\left(\right.$ see $\left.\left[\mathrm{JW}_{2}, \S 2\right]\right)$ with $\bar{A}=\left(\frac{\bar{a}, \bar{b}}{\bar{V}}\right)$ so the proposition shows $A$ is an inertial invariant valuation ring and so $S$ is a division ring.

The following examples make heavy use of property (4) of $\S 1$ : if $A$ is a Dubrovin valuation ring of a central simple algebra $S$ and $\mathscr{B}$ is a Dubrovin valuation ring of $\bar{A}$ then $B=\pi_{A}^{-1}(\mathscr{B})$ is a Dubrovin valuation ring of $S$, where $\pi_{A}: A \rightarrow \bar{A}$ is the canonical projection. If $F=Z(S), V=B \cap F$ and $W=A \cap F$, then $\widetilde{V}=V / J(W)$ is a valuation ring of $\bar{W}$. Following [ $\left.\mathrm{W}_{2}\right]$ we set $l_{B, A}$ equal to the number of extensions of $\widetilde{V}$ to $Z(\bar{A})$.

Example 4.2. $D, D_{1}, D_{2} F$-central division algebras with $D=D_{1} \otimes_{F} D_{2}, B$, $B_{1}, B_{2}$ Dubrovin valuation rings in $D, D_{1}, D_{2}$ respectively lying over $V$ with $B_{1}$ integral, $\Gamma_{B_{1}} \cap \Gamma_{B_{2}}=\Gamma_{V}$ and $\overline{B_{1}} \otimes_{\bar{V}} \overline{B_{2}}$ simple Artinian, but $\bar{B} \neq \overline{B_{1}} \otimes_{\bar{V}} \overline{B_{2}}$.

Proof. Let

$$
F=\mathbf{Q}(x, y), \quad D_{1}=\left(\frac{-3, x+y}{F}\right), \quad D_{2}=\left(\frac{-1, x}{F}\right)
$$

and $D=D_{1} \otimes_{F} D_{2}$. If $X$ is the composite of the $x$-adic valuation ring of $\mathbf{Q}(y)(x)$ with the $y$-adic valuation ring of $\mathbf{Q}(y)$ and $v$ is the corresponding valuation then (since $[\bar{X}(\sqrt{-3}, \sqrt{-1}): \bar{X}]=4$ and $v(x+y)=(1,0), v(x)=$ $(0,1))$; by $\left[\mathrm{JW}_{2}\right.$, Ex. 4.3] $D$ is a division ring.

Let $U=\mathbf{Q}(x)[y]_{(y)}$, the $y$-adic valuation ring of $F$ and $W$ the composite of $U$ with the $x$-adic valuation ring on $\mathbf{Q}(x)=\bar{U}$. So $\bar{W}=\mathbf{Q}$. Let $V$ be the 
composite of $W$ and the 5-adic valuation ring on $\mathbf{Q}$. Thus we have

$$
\begin{aligned}
\Gamma_{U} & =\mathbf{Z}, & \bar{U} & =\mathbf{Q}(x), \\
\Gamma_{W} & =\mathbf{Z} \times \mathbf{Z}, & \bar{W} & =\mathbf{Q}, \\
\Gamma_{V} & =\mathbf{Z} \times \mathbf{Z} \times \mathbf{Z}, & \bar{V} & =\mathbf{Z} / 5 \mathbf{Z} .
\end{aligned}
$$

In $D_{1}$, again by [JW 2, Ex. 4.3], $V$ extends to a nicely semiramified valuation ring $B_{1}$, as -3 is not a square mod 5 and $x+y$ does not have even value in $\Gamma_{V}$. Thus $B_{1}$ is integral (and defectless). For $B_{2}$, we first see that $W$ extends to a nicely semiramified valuation ring $A_{2} \subset D_{2}$ with $\overline{A_{2}}=\mathbf{Q}(\sqrt{-1}) . V / J(W)$ (the 5-adic valuation ring of $\mathbf{Q}$ ) extends in two ways to $\mathbf{Q}(\sqrt{-1})$. If $\mathscr{B}_{2}$ is one of these extensions then $B_{2}=\pi_{A_{2}}^{-1}\left(\mathscr{B}_{2}\right)$ is an extension of $V$ to $D_{2}$. Thus we have $\overline{B_{2}}=\overline{\mathscr{B}_{2}}=\mathrm{Z} / 5 \mathrm{Z}=\bar{V}$. Since $l_{B_{2}, A_{2}}=2$ it follows from [ $\left[\mathrm{W}_{2}\right.$, Theorem C] that $n_{B_{2}}=2$ and $\Gamma_{B_{2}}=\Gamma_{V}$. Therefore $\Gamma_{B_{1}} \cap \Gamma_{B_{2}}=\Gamma_{V}$ and $\overline{B_{1}} \otimes_{V} \overline{B_{2}}=\overline{B_{1}}$ is simple.

To construct $B$ we start with $U$. By Proposition 4.1, $U$ extends to inertial valuation rings $C_{i} \subset D_{i}$ with $\overline{C_{1}}=\left(\frac{-3, x}{\mathbf{Q}(x)}\right)$ and $\overline{C_{2}}=\left(\frac{-1, x}{\mathbf{Q}(x)}\right)$. By Theorem 3.4 (or [ $\mathrm{W}_{2}$, Proposition 3.3]) $C=C_{1} \otimes_{U} C_{2}$ is Dubrovin over $U$ in $D$ with $\bar{C}=\overline{C_{1}} \otimes_{\bar{U}} \overline{C_{2}}=M_{2}\left(\left(\frac{3, x}{\mathrm{Q}(x)}\right)\right) . V / J(U)$ (the composite of the $x$-adic valuation ring of $\mathbf{Q}(x)$ with $\mathbf{Z}_{(5)}$ ) extends to a nicely semiramified valuation ring $\mathscr{B}$ in $\left(\frac{3, x}{\mathrm{Q}(x)}\right)$. Let $B=\pi_{C}^{-1}\left(M_{2}(\mathscr{B})\right)$. Then $B \subset D$ is (integral) Dubrovin over $V$ with $\bar{B}=M_{2}(\overline{\mathscr{B}})=M_{2}((\mathrm{Z} / 5 \mathrm{Z})(\sqrt{3}))=M_{2}\left(\overline{B_{1}}\right)$. Therefore $\bar{B} \neq \overline{B_{1}} \otimes_{\bar{V}} \overline{B_{2}}=$ $\overline{B_{1}}$.

This shows the need for both $B_{1}$ and $B_{2}$ to be integral in Theorem 3.3. Notice also in the above example that $U$ (and $W$ ) does not extend to an invariant valuation ring of $D$ even though it does so extend to both $D_{1}$ and $D_{2}$.

The next example shows that the tensor product of two central simple algebras need not contain an integral Dubrovin valuation ring even though each piece does. This example shows the necessity for assuming the simplicity of $\overline{B_{1}} \otimes_{V} \overline{B_{2}}$ in Theorem 3.3. The example in $\left[\mathrm{M}_{1}\right]$ shows the need of defectlessness in at least one of the factors.

Example 4.3. $D, D_{1}, D_{2} F$-central division algebras with $D=D_{1} \otimes_{F} D_{2}$, $B_{i} \subset D_{i}$ integral Dubrovin valuation rings over $V$ with $\Gamma_{B_{1}} \cap \Gamma_{B_{2}}=\Gamma_{V}$ but no Dubrovin valuation ring of $D$ lying over $V$ is integral.

Proof. Let

$$
F=\mathbf{Q}((x))((y)), \quad D_{1}=\left(\frac{x, 3}{F}\right), \quad D_{2}=\left(\frac{y,-3}{F}\right)
$$

and $D=D_{1} \otimes_{F} D_{2} . \quad D$ will be shown to be a division ring in much the same manner as in the previous example. Let $U$ be the $y$-adic valuation ring 
of $F, W=\mathbf{Q}[[x]]+y \mathbf{Q}((x))[[y]]$, the usual rank 2 valuation ring of $F$ with $\bar{W}=\mathbf{Q}$, and $V$ the composite of $W$ and the 5-adic valuation ring of Q. Notice that $U$ and $W$ are Henselian but $V$ is not. By Proposition 4.1 and [JW ${ }_{2}$, Ex. 4.3], $U$ extends to an inertial valuation ring $C_{1} \subset D_{1}$ with $\overline{C_{1}}=\left(\frac{x, 3}{\mathrm{Q}((x))}\right)$ and to a nicely semiramified extension $C_{2} \subset D_{2}$ with $\Gamma_{C_{2}}=\frac{1}{2} \mathbf{Z}$ and $\overline{C_{2}}=\mathbf{Q}((x))(\sqrt{-3})$. Hence $D_{1}$ and $D_{2}$ are division rings. We show that $\overline{C_{1}} \otimes_{\bar{U}} \overline{C_{2}}=\left(\frac{x, 3}{\mathrm{Q}((x))(\sqrt{-3})}\right)$ is a division ring, which by Corollary 3.5 shows that $D$ is a division ring and $U$ extends to an invariant valuation ring $C \subset D$ with $\Gamma_{C}=\frac{1}{2} \mathbf{Z}$ and $\bar{C}=\overline{C_{1}} \otimes_{\bar{U}} \overline{C_{2}}$. To see this, since $\sqrt{3} \notin \mathbf{Q}((x))(\sqrt{-3})$, with respect to the $x$-adic valuation $\left(\frac{x, 3}{Q((x))(\sqrt{-3})}\right)$ is a nicely semiramified division algebra, by [ $\mathrm{JW}_{2}$, Ex. 4.3]. Hence $D$ is a division ring and $U$ extends to an invariant valuation ring $C \subset D$ with $\Gamma_{C}=\frac{1}{2} \mathbf{Z}$ and $\bar{C}=\overline{C_{1}} \otimes_{\bar{U}} \overline{C_{2}}$.

Using [ $\mathrm{JW}_{2}$, Ex. 4.3] again we see that $W$ extends to nicely semiramified valuation rings $A, A_{1}, A_{2} \subset D, D_{1}, D_{2}$ respectively with

$$
\begin{aligned}
\Gamma_{A} & =\frac{1}{2} \mathbf{Z} \times \frac{1}{2} \mathbf{Z}, & \bar{A} & =\mathbf{Q}(\sqrt{3}, \sqrt{-3}), \\
\Gamma_{A_{1}} & =\frac{1}{2} \mathbf{Z} \times \mathbf{Z}, & \overline{A_{1}} & =\mathbf{Q}(\sqrt{3}), \\
\Gamma_{A_{2}} & =\mathbf{Z} \times \frac{1}{2} \mathbf{Z}, & \overline{A_{2}} & =\mathbf{Q}(\sqrt{-3}) .
\end{aligned}
$$

The 5-adic valuation ring $\mathbf{Z}_{(5)}$ extends uniquely to $\mathbf{Q}(\sqrt{3})$ and $\mathbf{Q}(\sqrt{-3})$ but extends in two ways to $\mathbf{Q}(\sqrt{3}, \sqrt{-3})=\mathbf{Q}(\sqrt{3}, \sqrt{-1})$ as 3 is not a square mod 5 but -1 is. Let $T_{i}$ be the unique extension of $\mathrm{Z}_{(5)}$ to $\overline{A_{i}}$ and $T$ one of the extensions to $\bar{A}$. Let $B, B_{1}, B_{2}$ be the composite of $A, A_{1}, A_{2}$ with $T$, $T_{1}, T_{2}$ respectively. We then have $l_{B_{1}, A_{1}}=l_{B_{2}, A_{2}}=l_{A_{1}, C_{1}}=l_{A_{2}, C_{2}}=1$ and $l_{B, A}=2, l_{A, C}=1$. Thus using [ $\mathrm{W}_{2}$, Theorem D], $s_{B_{1}}=s_{B_{2}}=1$ but $s_{B}=2$. Hence $B_{1}$ and $B_{2}$ are integral but $B$ is not. Since \pm 3 is not a square mod 5, by [JW 2 , Ex. 4.3] $B_{1}$ and $B_{2}$ are nicely semiramified valuation rings with $\Gamma_{B_{1}}=\mathbf{Z} \times \frac{1}{2} \mathbf{Z} \times \mathbf{Z}, \Gamma_{B_{2}}=\mathbf{Z} \times \mathbf{Z} \times \frac{1}{2} \mathbf{Z}, \overline{B_{1}}=(\mathbf{Z} / 5 \mathbf{Z})(\sqrt{3}), \overline{B_{2}}=(\mathbf{Z} / 5 \mathbf{Z})(\sqrt{-3})=$ $(\mathbf{Z} / 5 \mathrm{Z})(\sqrt{3})=\overline{B_{1}}$. Thus $\Gamma_{B_{1}} \cap \Gamma_{B_{2}}=\Gamma_{V}$ and $\overline{B_{1}} \otimes_{\bar{V}} \overline{B_{2}}$ is not simple. Hence the simplicity of the ring $\frac{B_{1}}{B_{1}} \otimes_{\bar{V}} \frac{B_{2}}{B_{2}}$ is needed in Theorem 3.3.

\section{REFERENCES}

[A] S. A. Amitsur, On central division algebras, Israel J. Math. 12 (1972), 408-420.

[Be] H. Benz, Über eine Bewertungstheorie der Algebren und ihre Bedeutung für die Arithmetik, Schriftenreihe der Inst. für Math., Heft 9, Berlin, 1961.

[B] N. Bourbaki, Commutative algebra, Chapter 6, Valuations, Hermann, Paris, 1961.

[BG] H. H. Brungs and J. Gräter, Extensions of valuation rings in central simple algebras, Trans. Amer. Math. Soc. (to appear).

[Dr] P. Draxl, Ostrowski's theorem for Henselian valued skew fields, J. Reine Angew. Math. 354 (1984), 213-218.

[DK] P. Draxl and M. Kneser, $S_{1}$ von Schiefkörpern, Lecture Notes in Math., vol. 778, SpringerVerlag, Berlin, Heidelberg and New York, 1980. 
[D 1 ] N. I. Dubrovin, Noncommutative valuation rings, Trudy Moskov. Mat. Obshch. 45 (1982), 265-280; English transl., Trans. Moscow Math. Soc. 45 (1984), 273-287.

[D $\left.\mathrm{D}_{2}\right]$ _ Noncommutative valuation rings in simple finite-dimensional algebras over a field, Mat. Sb. 123 (165) (1984), 496-509; English transl., Math. USSR-Sb. 51 (1985), 493-505.

[E] O. Endler, Valuation theory, Springer-Verlag, New York, 1972.

[JW 1 ] B. Jacob and A. Wadsworth, A new construction of noncrossed product algebras, Trans. Amer. Math. Soc. 293 (1986), 693-721.

[JW 2 ] _ Division algebras over Henselian fields, J. Algebra (to appear).

[J] N. Jacobson, PI-algebras, an introduction, Lecture Notes in Math., vol. 441, Springer-Verlag, Berlin, Heidelberg and New York, 1975.

[K] A. Kupferoth, Valuated division algebras and crossed products, J. Algebra 108 (1987), 139-150.

[L] T.-Y. Lam The algebraic theory of quadratic forms, Benjamin, Reading, Mass., 1973.

[M $\mathrm{M}_{1}$ ] P. Morandi, The Henselization of a valued division algebra, J. Algebra 122 (1989), 232-243.

$\left[\mathrm{M}_{2}\right]$ - Valuation rings in division rings and central simple algebras, Doctoral Dissertation, Univ. of California, San Diego, 1988.

[MW] P. Morandi and A. Wadsworth, Integral Dubrovin valuation rings, Trans. Amer. Math. Soc. 315 (1989), 623-640.

[P] V. P. Platonov, The Tannaka-Artin problem and reduced $K$-theory, Izv. Akad. Nauk SSSR Ser. Mat. 40 (1976), 227-261; English transl., Math. USSR-Izv. 10 (1976), 211-243.

[PY] V. P. Platonov and V. I. Yanchevskii, Dieudonnês conjecture on the structure of unitary groups over a division ring, and hermitian $K$-theory, Izv. Akad. Nauk SSSR Ser. Mat. 48 (1984), 1266-1294; English transl., Math. USSR-Izv. 25 (1985), 573-599.

[R] I. Reiner, Maximal orders, Academic Press, London, 1975.

[S] O. F. G. Schilling, The theory of valuations, Math Surveys, no. 4, Amer. Math. Soc., Providence, R.I., 1950.

[T] J.-P. Tignol, Cyclic and elementary abelian subfields of Malcev-Neumann division algebras, J. Pure Appl. Algebra 42 (1986), 199-220.

[TA] J.-P. Tignol and S. A. Amitsur, Kummer subfields of Malcev-Neumann division algebras, Israel J. Math. 50 (1985), 114-144.

[TW] J.-P. Tignol and A. Wadsworth, Totally ramified valuations on finite dimensional division algebras, Trans. Amer. Math. Soc. 302 (1987), 223-250.

[V] J. Van Geel, Places and valuations in noncommutative ring theory, Lecture Notes in Pure and Appl. Math., no. 71, Dekker, New York, 1981.

[W 1 ] A. Wadsworth, Dubrovin valuation rings, Proceedings of the NATO Workshop "Perspectives in Ring Theory" (F. van Oystaeyen and L. Le Bruyn, eds.), Antwerp, 1988, pp. 359-374.

$\left[\mathrm{W}_{2}\right]$ _ Dubrovin valuation rings and Henselization, Math. Ann. 283 (1989), 301-328.

Department of Mathematics, University of California, San Diego, la Jolla, CaliforNIA 92093

Current address: Department of Mathematics, University of Texas, Austin, Texas 78712 